\title{
Ecografía ocular para la estimación de la presión intracraneal: estudio de la factibilidad de la técnica
}

\section{Ocular ultrasound to estimate intracranial pressure: feasibility of the technique}

Jhonelle Waissbluth', María Carolina Cabrera Schulmeyer ${ }^{1}$,

\begin{abstract}
Intracranial pressure (ICP) is a parameter of high clinical relevance. Today invasive methods are reference for its measurement. The diameter of the optic nerve sheath (NOSD) is influenced by changes in ICP. A NOSD higher than 5 $\mathrm{mm}$ is well correlated with a ICP over $20 \mathrm{mmHg}$. Objective: To evaluate the feasibility in measuring NOSD by ultrasound (ECO) made by an anesthesiologist. The secondary objective is to assess the impact of the Trendelenburg position in the NOSD. Material and Methods: A descriptive study was carried out between April-September 2014, with the participation of 89 volunteers, ages 20 to 39 years old, 49 men, without fasting, with defined inclusion criteria to ensure absence of underlying disease, chronic drugs intake and normovolemic state. After application of gel, a linear transducer $(10-15 \mathrm{MHz})$ was positioned on the eyeball to measure the NOSD; measurements were performed with $0^{\circ}$ tilt and then after five minutes in $45^{\circ}$. Statistical analysis was performed with STATA 10.0, with $p$-value $<0,05$ for statistical significance. Results: The procedure was well tolerated. Echocardiograph windows obtained allowed measurements in all cases with an average NOSD of $2.3 \pm 6 \mathrm{~mm}$ at $0^{\circ}$ and $2,4 \pm 0,8 \mathrm{~mm}$ at $45^{\circ}(p=$ 67). Conclusion: In this group of patients, young, healthy, without underlying disease, not undergoing anesthesia and outside the operating room, the use of ocular ECO allowed measurements of DVNO in a simple and reproducible way, without adverse effects. The existence of this tool, that estimates the PIC indirectly, is of great anesthesiology utility.
\end{abstract}

Key words:

Intracranial pressure, optic nerve, ultrasound

Departamento de Anestesiología, Universidad de Valparaíso, Sede Hospital Clínico de la Fuerza Aérea, Santiago, Chile.

Fecha de recepción: 30 de abril de 2018

Fecha de aceptación: 20 de mayo de 2018

ORCID

https://orcid.org/0000-0002-6105-5780

Correspondencia:

Dra. María Carolina Cabrera Schulmeyer

Email: maria.cabrera@uv.cl 


\section{RESUMEN}

La presión intracraneal (PIC) es un parámetro de alta relevancia clínica. En la actualidad los métodos invasivos son su referencia para medición. El diámetro de la vaina del nervio óptico (DVNO) medida con ecografía se ve influenciado por cambios en la PIC. Se ha demostrado que el DVNO superior a $5 \mathrm{~mm}$ se correlaciona con PIC mayor que $20 \mathrm{mmHg}$. Objetivo: Evaluar la factibilidad en la medición del DVNO mediante ecografía (ECO) realizada por anestesiólogos. Como objetivo secundario, evaluar el impacto de la posición de Trendelenburg (PT) en el DVNO. Material y Métodos: Se realizó estudio descriptivo, entre abril-octubre 2014, con la participación de 89 voluntarios, entre 20 y 39 años, 49 hombres, sin ayuno, con criterios de inclusión definidos para asegurar inexistencia de patología de base, ingesta crónica de fármacos y estado de normovolemia. Tras aplicación de gel se posicionó un transductor linear (10-15 Mhz) sobre el globo ocular para la medición del DVNO; las mediciones fueron realizadas con $0^{\circ}$ de inclinación y luego tras cinco minutos en PT. El análisis estadístico fue realizado con STATA 10.0, valor- $p<0,05$ para significancia estadística. Resultados: El procedimiento fue tolerado de manera adecuada. Las ventanas ecográficas obtenidas permitieron realizar mediciones en todos los casos con valor de DVNO promedio de $2,3 \pm 0,6 \mathrm{~mm}$ a $0^{\circ}$ y $2,4 \pm 0,8 \mathrm{~mm}$ a $45^{\circ}(\mathrm{p}=67)$, sin diferencia estadística. Conclusión: En este grupo de pacientes, jóvenes, sanos, sin patología de base, no sometidos a anestesia y en el contexto "fuera de pabellón", la realización de la ECO ocular permitió la medición del DVNO de manera sencilla, reproducible y sin efectos adversos. La existencia de esta herramienta que estima la PIC de manera indirecta puede ser de gran utilidad anestesiológica.

\section{Palabras clave:}

Presión intracraneal, nervio óptico, ecografía

\section{Introducción}

E I ultrasonido (US) ha ingresado con mucha fuerza al quirófano y a la práctica anestesiológica diaria, describiéndose cada vez más utilizaciones para evaluar distintos parénquimas como ECO pulmonar, traqueal, vesical, de grandes vasos y ocular.

EI US para evaluar la presión intracraneana (PIC) es una técnica sencilla de realizar, segura, reproducible y comparable a otras técnicas invasivas. Existen distintos métodos invasivos para la medición de la PIC. Actualmente, el método de referencia es la medición mediante la instalación de catéteres intraventriculares[1], con sus respectivas infecciosas complicaciones por corresponder a un método invasivo.

Con respecto al uso de ecografía para evaluación de la vaina del nervio óptico (DVNO), ésta sería muy útil como predictor de la PIC. Es un método no invasivo, fácil de aprender, reproducible, dinámico, de bajo costo económico, se puede realizar "al lado de la cama del paciente" sin la necesidad de cirujano o pabellón, y presenta baja frecuencia de complicaciones.

El NO se encuentra envuelto por una vaina (leptomeninges) y se continúa con la duramadre que es a la capa más externa de las meninges. El compartimento subaracnoideo corresponde a la distancia entre el nervio y su vaina y en situación basal mide $0,1 \mathrm{~mm}$. En su interior fluye líquido cefalorraquídeo (LCR) y una red de trabéculas, septos y pilares interpuestos entre la aracnoides y la duramadre, con mayor densidad hacia posterior. El LCR baña por completo al NO y se encuentra en comunicación directa con el resto de los espacios y cisternas que lo contienen, creando así una presión homogénea. Cuando se genera un aumento de la PIC, el LCR se desplaza al espacio perineural, generando un aumento de presión del NO y desplazamiento de su vaina. El valor máximo del DVNO es 4,9 $\mathrm{mm}$. Valores sobre $5 \mathrm{~mm}$ a la ECO se correlacionan con una PIC mayor a $20 \mathrm{mmHg}$, con una sensibilidad de $86 \%$, especificidad de $98 \%$, valor predictivo positivo de $92 \%$ y valor predictivo negativo $96 \%$ [2]. Para la medición exacta del DVNO el método de referencia es la resonancia nuclear magnética. Baüerle et al[3] en su estudio, evaluaron la reproductibilidad y exactitud del método ecográfico en relación a la RNM demostrando correlación entre ambos, con Coeficiente de Correlación de Pearson $r=0,72$.

La posición de Trendelenburg (PT) consiste en la 
posición decúbito supino sobre una mesa inclinada a $45^{\circ}$ grados, con la cabeza hacia el suelo. Ésta fue descrita como una técnica que mejora el gasto cardíaco mediante aumento de la pre-carga cardiaca y se evaluará si influye en el DVNO.

El objetivo primario de este trabajo fue evaluar la factibilidad en la medición del DVNO mediante ecografía (ECO) realizada por anestesiólogos, en voluntarios jóvenes y sanos. El objetivo secundario fue evaluar eventuales diferencias en el DVNO tras cinco minutos en PT en el mismo grupo de voluntarios.

\section{Material y Métodos}

Se realizó un estudio observacional, de tipo descriptivo, entre los meses de abril y septiembre del año 2014. Este tipo de estudio fue elegido dado que la pregunta del investigador fue la factibilidad de la técnica en un grupo particular de personas, sin asignación de exposición alguna por parte de los investigadores, sin intención de correlación alguna y sin grupo de comparación entre los sujetos estudiados.

Se reclutó a voluntarios, de ambos sexos, entre 20 y 39 años de edad. Con ello se disminuyó la probabilidad de existencia de condiciones clínicas que pudiesen alterar las mediciones basales del NO y su vaina. Se obtuvo previa aprobación del Comité de Ética para la realización del estudio y consentimiento informado firmado por cada voluntario. Los voluntarios fueron citados al hospital FACH, sin ayuno, donde se aplicaron criterios elaborados por los investigadores para asegurar inexistencia de patología de base, ingesta crónica de fármacos y estado de normovolemia. El procedimiento fue llevado a cabo en la Sala de Recuperación Quirúrgica del Hospital FACH, en donde cada voluntario fue monitorizado con registro de presión arterial, saturometría de pulso y frecuencia cardíaca de manera no invasiva. La ECO fue realizada por un solo operador anestesiólogo con entrenamiento especial en ultrasonido de superficie, con la utilización de un equipo Micromaxx Sonosite turbo con transductor lineal (11-15 Mhz). En cada uno de los voluntarios se evaluó la existencia de adecuadas ventanas ecográficas para la realización de mediciones. Cada voluntario fue recostado sobre una camilla con $0^{\circ}$ de inclinación. La ventana utilizada fue el ojo, con previa aplicación de una fina capa de gel, el transductor fue colocado sobre el párpado superior de un ojo estando éste cerrado. Se ubicó la parte anterior del NO en su plano axial y se midió el DVNO desde sus bordes internos, aproximadamente a $3 \mathrm{~mm}$ de la papila (Figura 1). Luego se aplicó PT durante 5 minutos y se realizaron las mismas mediciones en el mismo ojo. Valores de presión arterial, saturometría de pulso, frecuencia cardiaca y DVNO fueron registros en decúbito supino con $0^{\circ}$ inclinación y tras 5 minutos en PT para posterior análisis.

El análisis de la base de datos fue realizado por un profesional en bioestadística, ciego al estudio, con la utilización del programa computacional STATA versión 10.0 IC-inter cooled, el cual corresponde a un programa estadístico de amplio uso en la investigación clínica. Dada las características de este estudio y el tipo de pregunta de investigación, se utilizó el valor-p como indicador de significancia estadística con valor de corte $<0,05$, con la finalidad de medir la probabilidad de que los resultados obtenidos hayan sido producto del azar.

\section{Resultados}

De un total de 89 voluntarios sanos, 50 eran mujeres y 39 hombres. La edad promedio de los voluntarios fue de $31 \pm 5$ años. No hubo pérdida de sujetos durante la investigación (Tabla 1).

En cuanto al resultado primario, el procedimiento fue tolerado de manera adecuada por los voluntarios, sin efectos adversos inherentes a la técnica. Las ventanas ecográficas obtenidas permitieron la realización de mediciones en todos los casos. El diámetro del NO fue en promedio 2,3 $\pm 0,6 \mathrm{~mm}$ (rango 1,9 a 2,5), sin diferencias estadísticamente significativas entre sexos y edades límites.

En cuanto al resultado secundario, el DVNO a $0^{\circ}$ de inclinación fue en promedio 2,2 $\pm 0,6 \mathrm{~mm}$, y luego tras cinco minutos en PT fue en promedio 2,3 $\pm 0,5$

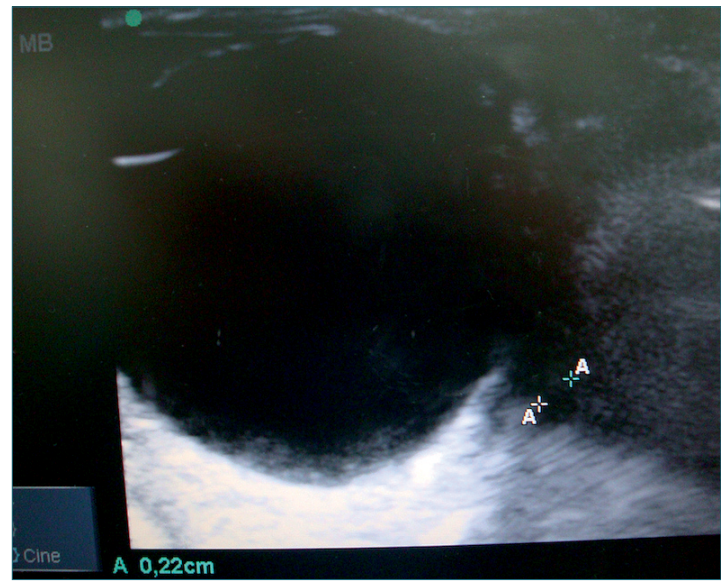

Figura 1. Ultrasonido del globo ocular, la medición muestra el diámetro de la vaina del nervio óptico. 


\begin{tabular}{lcc}
\multicolumn{2}{c}{$\begin{array}{c}\text { Tabla 1. Características de la población } \\
\text { estudiada }\end{array}$} \\
\hline & $\begin{array}{c}\text { Masculino } \\
\mathbf{n}=\mathbf{3 9}\end{array}$ & $\begin{array}{c}\text { Femenino } \\
\mathbf{n}=\mathbf{5 0}\end{array}$ \\
& $22 \pm 8$ & $21 \pm 8$ \\
Edad & $78 \pm 10$ & $63 \pm 5$ \\
Peso & $1,69 \pm 9$ & $1,62 \pm 7$ \\
Talla & & \\
\hline
\end{tabular}

$\mathrm{mm}$. Esta diferencia no fue estadísticamente significativa (valor-p $=0,76$ ). Resultado de otros parámetros evaluados se detallan en Tabla 2.

\section{Discusión}

En este estudio se demostró que la ecografía ocular es una técnica factible de realizar, sencilla y segura. Una de sus principales ventajas está en que no es invasiva y puede ser realizada "al lado de la cama del paciente".

Los valores del diámetro de la vaina del nervio óptico fueron todos normales no pesquisándose casos de aumento de PIC, como era en esta serie.

Desde hace más de 15 años la relación entre mediciones del NO y cambios en la PIC ha sido estudiada. En 1993, Liu et al[4] evaluaron la relación entre distintos valores de PIC y los correspondientes DVNO. El estudio demostró la existencia de mayor DNVO en pacientes con aumento de la PIC con valor-p $<0,0001$, concluyendo que el DVNO es dinámico y varía ante cambios en la PIC.

Heidi et al.[5], realizaron un estudio observacional, prospectivo, en 20 pacientes que presentaban ventriculostomía. Demostraron la existencia de correlación entre DVNO > $5 \mathrm{~mm}$ y $\mathrm{PIC}>20 \mathrm{mmHg}$ con valor-p $<0,0005$, sensibilidad y especificidad de $88 \%$ y $93 \%$ respectivamente.

Geeraerts et al.[6], introdujeron la utilización de la RNM para la medición del DVNO. Ellos demostraron que éste diámetro era significativamente mayor en pacientes con hipertensión endocraneana (HTE).

Como se mencionó anteriormente, se ha objetivado una correlación entre la ECO y el método de referencia para la medición del DVNO.

Tras analizar los resultados obtenidos en este estudio se puede afirmar que, en este grupo de pacientes, jóvenes, sanos, sin patología de base, no sometidos a anestesia y en el contexto fuera de pabellón, la realización de la ECO ocular permitió la medición del DVNO de manera sencilla y reproducible. Se demostró

\begin{tabular}{|c|c|c|c|}
\hline Parámetro & $\begin{array}{c}0^{\circ} \\
\text { inclinación }\end{array}$ & $\begin{array}{c}45^{\circ} \\
\text { inclinación }\end{array}$ & Valor de $p$ \\
\hline $\begin{array}{l}\text { PANI } \\
(\mathrm{mmHg})\end{array}$ & $76 \pm 12$ & $78 \pm 11$ & 0,7 \\
\hline FC (Ipm) & $72 \pm 7$ & $75 \pm 4$ & 0,8 \\
\hline $\begin{array}{l}\text { Saturación } \\
(\%)\end{array}$ & $97 \%$ & $97 \%$ & 1 \\
\hline
\end{tabular}

PANI = presión arterial media; $F C$ = frecuencia cardiaca; $45^{\circ}$ inclinación corresponde a mediciones tras cinco minutos en PT.

además, que no existió una variación estadísticamente significativa del DVNO tras cinco minutos en PT.

La ausencia de diferencia entre el DVNO a $0^{\circ}$ y a $45^{\circ}$ puede explicarse porque el paciente está despierto y no ha sido sometido a intervención anestésica alguna, por lo que su tono y reflejo vasomotores están conservados. Será interesante evaluar lo que ocurre en pacientes sometidos a anestesia general.

La medición del DVNO mediante ECO ocular puede ser gran utilidad clínica ya que una técnica que permite la obtención de diagnósticos de manera rápida, es de bajo costo económico y altamente accesible ya que la mayoría de los quirófanos cuentan con estos equipos. Al ser un método de monitorización no invasiva se encuentra casi exento de complicaciones por lo que puede realizarse de manera repetida sin riesgos para el paciente. Es un recurso al "lado de la cama" para pacientes que por distintos motivos no pueden ser movilizados a RNM o pabellón. Su curva de aprendizaje contiene un número de personas muy reducido, llegando a ser de diez procedimientos para personas con experiencia en ecografía de superficie básica, y de veinte para los que no la tienen, aunque en nuestra experiencia fue de un número menor aún.

Sus limitaciones se manifiestan con mayor frecuencia en pacientes con trauma ocular, enfermedad del nervio óptico y neuropatía traumática secundaria a TEC. La presencia de falsos positivos ha sido registrada en algunos casos de tumores, pseudotumores, metástasis, inflamación, hemorragia del NO, enfermedad de Graves y Sarcoidosis[7].

Para finalizar, cabe mencionar que este estudio ha sido el paso inicial para responder una serie de interrogantes respecto a este tema. Sería de gran interés continuar este tipo de investigación, de medición del DVNO mediante ECO, en pacientes que se encuentren bajo el efecto de anestesia general, o casos en que se sospecha HTE. 


\section{Referencias}

1. Evaluation and management of intracranial pressure in adults. Sepideh Amin-Hanjani. 2013 Jul.

2. Kimberly HH, Shah S, Marill K, Noble $\mathrm{V}$. Correlation of optic nerve sheath diameter with direct measurement of intracranial pressure. Acad Emerg Med. 2008 Feb;15(2):201-4.

3. Bäuerle J, Schuchardt F, Schroeder L, Egger K, Weigel M, Harloff A. Reproducibility and accuracy of optic nerve sheath diameter assessment using ultrasound compared to magnetic resonance imaging. BMC Neurol. 2013 Dec;13(1):187.

4. Newman WD, Hollman AS, Dutton GN, Carachi R. Measurement of optic nerve sheath diameter by ultrasound: a means of detecting acute raised intracranial pressure in hydrocephalus. Br J Ophthalmol. 2002 Oct;86(10):1109-13.

5. Geeraerts T, Newcombe VF, Coles JP, Abate MG, Perkes IE, Hutchinson PJ, et al. Use of T2-weighted magnetic resonance imaging of the optic nerve sheath to detect raised intracranial pressure. Crit Care. 2008;12(5):R114.

6. Soldatos T, Chatzimichail K, Papathanasiou M, Gouliamos A. Optic nerve sonography: a new window for the non-invasive evaluation of intracranial pressure in brain injury. Emerg Med J. 2009 Sep;26(9):630-4.

7. Raboel PH, Bartek J Jr, Andresen $\mathrm{M}$, Bellander BM, Romner B. Intracranial Pressure Monitoring: Invasive versus Non-Invasive Methods-A Review. Crit Care Res Pract. 2012;2012:950393. 